\title{
Metamaterials and metaoptics
}

\author{
Ross C. McPhedran', Ilya V. Shadrivov², Boris T. Kuhlmey and Yuri S. Kivshar2* \\ University of Sydney and Australian National University, Australia
}

\begin{abstract}
We discuss the topics of metamaterials-electromagnetic composites offering simultaneous control of electric and magnetic fields through structuring on a fine scale compared with the wavelength of light-and metaoptics, including striking optical effects achieved using metamaterial-based systems such as backward wave propagation and negative refraction. We survey fabrication methods and past achievements for systems working at long wavelengths and near-visible wavelengths, with emphasis on the recent development of this field in Australia. We select several striking conceptual and technological advances made in this rapidly developing field, such as cloaking, nonlinear metamaterials and drawable fiber-based metamaterials.
\end{abstract}

$\mathrm{T}$ he idea of a new class of media that has unusual properties with respect to electromagnetic wave propagation is generally attributed to the Russian physicist Victor Veselago [1]. He considered what would be the consequences if one could create or find materials for which the dielectric permittivity $\varepsilon$ and the magnetic permeability $\mu$ were both negative. He deduced that one striking outcome would be that the refractive index of the material would seem to be negative, causing the incident and refracted waves to lie on the same side of the normal to the interface between a 'standard' medium and the new medium. This phenomenon is now known as negative refraction (Figure 1(a)) and is associated with other phenomena in which the sign of the refractive index is reversed, such as Doppler shift, Cherenkov angle, Goos-Hänchen shift and radiation pressure. Another consequence of a negative refractive index is that the electric, magnetic and wave vectors form a left-handed triad, rather than the right-handed triad found in dielectrics. For this reason, such materials are called left-handed media, or sometimes double-negative media.

If one considers the conservation of momentum at the interface between a standard material and a left-handed metamaterial in which negative refraction is occurring (Figure 1(b)), two viewpoints are valuable. In the first, the metamaterial is regarded as a continuous medium characterized by an optical refractive index (or perhaps a permittivity tensor), and thus conservation of momentum requires the index to be negative. In the second, the metamaterial is regarded as being composed of discrete elements (perhaps at the level of atoms or molecules, or larger) separated by a vacuum. This means that left-handed metamaterials must have an internal structure whose strong interaction provides momentum transfer parallel to the interface, and also in the correct direction, much as a diffraction grating or crystal can. Hence, we require a well-designed structured material to provide this new behavior, and it should be structured on a scale much finer than the wavelength of incident light if it is to be regarded as a new type of solid, rather than just a diffraction grating or stack of diffraction gratings.

Related fields of science already exist in which structured materials are used to give desired optical properties. One of these is the study of composite materials, for which the most authoritative account may be found in a book by Milton [2]. This field dates back to Egyptian and Roman times, when different colors were given to glass by mixing chosen metallic particles into the glass matrix. Optical composite materials are

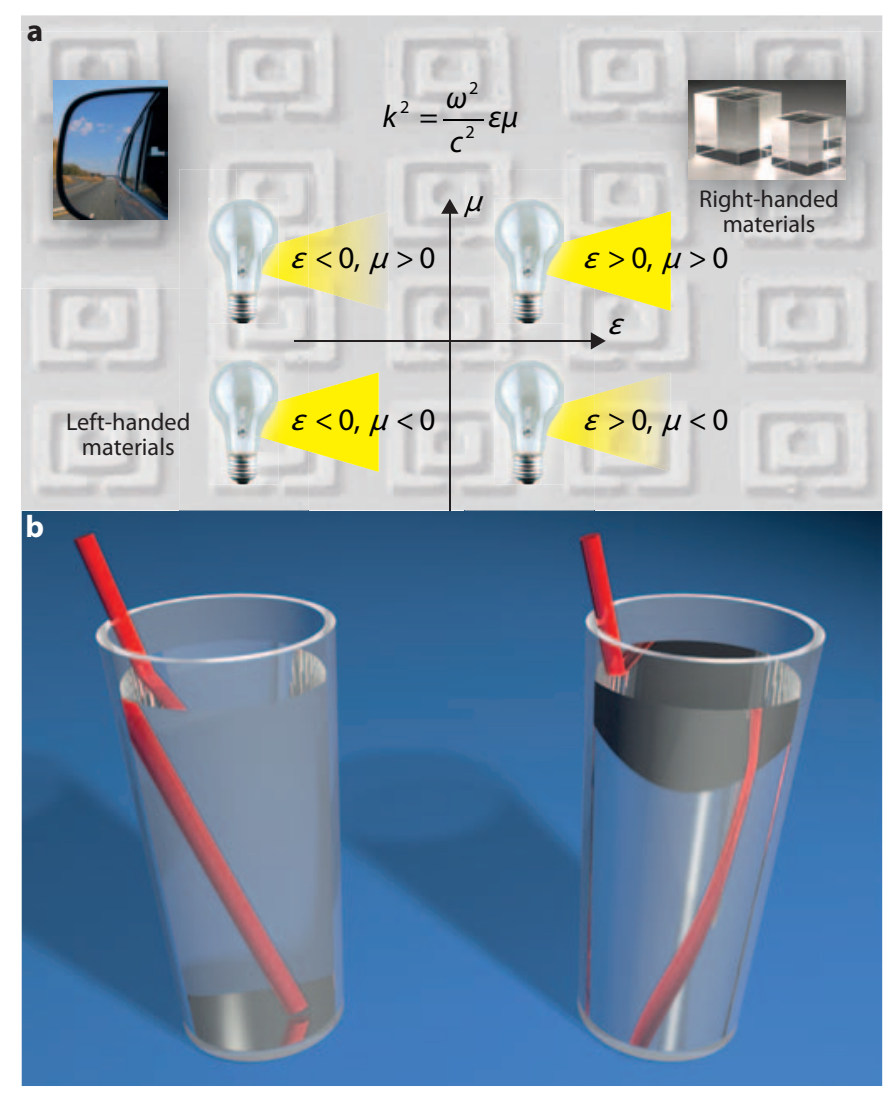

Figure 1. (a) The regions of possible permittivity $(\varepsilon)$ and permeability $(\mu)$, showing normal refraction (top right) and negative refraction (bottom left). (b) Normal refraction (left) and left-handed refraction (right).

generally used in the quasistatic limit, where the particle size is much finer than the wavelength of incident light. One can use electrostatics to calculate an effective dielectric permittivity or refractive index for the particle-matrix structure, and exploit the effective permittivity or 
refractive index in Maxwell's equations to describe the wave properties of the structured material. The particle-matrix structure is then regarded as an equivalent homogeneous medium-the structure is said to be homogenizable_-and the equivalent properties of the structure are said to have been arrived at by homogenization. The general rule of thumb is that composite structures are homogenizable if their spatial scale is 5-10 times smaller than the wavelength.

Plasmonics and photonic crystals are two other fields involving structured materials that are designed to control light propagation and interactions. Although negative refraction can be demonstrated in photonic crystals [3], one essential difference between metamaterials and the three related fields of composite materials, plasmonics and photonic crystals is that "metamaterials make light walk on both feet," as aptly noted by German physicist Martin Wegener. By this Wegener means that metamaterials incorporate elements that control magnetic fields as well as electric fields, typically through the use of resonances involving both types of field. With all these criteria in play (structuring much finer than the wavelength of light, the ability to induce both magnetic and electric resonances in the same wavelength range, and the requirement that both permittivity and permeability be negative), it is hardly surprising that Veselago's visionary proposal was neglected for more than 30 years. Around the turn of the millennium, conditions were beginning to come together to make an attack on the science of metamaterials. Fabrication technology had advanced to the point at which structuring materials on the $20-50 \mathrm{~nm}$ scale, although still difficult, was achievable. Sophisticated electromagnetic modeling packages were available that allowed daring ideas involving complicated geometries to be accurately simulated before fabrication was attempted. However, it took one more remarkable development to launch this new field: the famous paper by Sir John Pendry [4], which took Veselago's idea of negative refraction and applied it to construct, in principle, a flat lens capable of resolving well beyond the Rayleigh diffraction limit. The paper drew immediate critical reaction, but more importantly initiated a fever of activity, including experimental verifications [5] of negative refraction and numerous theoretical developments of the Pendry-Veselago geometry.

The second major phase of metamaterial research began with two papers published in the same issue of Science in 2005 [6,7]. These papers launched a new way of thinking about the design of electromagnetic systems, known as transformation optics, and introduced a new goal for designers: electromagnetic cloaking. Transformation optics applies the methods of general relativity to electromagnetism, giving concrete procedures for designing everything from sketched ray paths to spatially varying material properties capable of forcing light to follow a desired route. Researchers in the field of electromagnetic cloaking have the ambitious goal of designing systems that are undetectable to surveillance systems based on probe beams revealing the presence of objects. Both the perfect lens and the electromagnetic cloak became major research topics following the first experimental investigation demonstrating the feasibility of cloaking [8]. Before going on to cover further developments in metamaterials and metaoptics, we will pause to discuss some papers that may be viewed as precursors to the remarkable developments in these fields.

\section{The prehistory of metamaterials and metaoptics}

One characteristic of a left-handed medium is that the phase of a wave passing through it advances in a direction opposite to that of the energy flow. The study of such 'backward waves' may be traced back to at least 1904 (for a comprehensive account, see the web page by Moroz [9]). Lamb [10] may have been the first to suggest the possibility of a system (mechanical in his case) capable of supporting a wave whose group velocity is in the direction opposite to its phase velocity. Schuster [11] speculated as to whether it would be possible to translate Lamb's result to electromagnetic waves. Early influential works in Russian literature regarding this question are due to Mandelshtam [12] and Dolin [13].
Dolin's work may be the first in a number of papers in which the ideas of transformation optics were developed independently, to varying degrees of sophistication. Another important source of ideas has been the study of artificial dielectrics by electrical engineers, who are chiefly interested in the microwave properties of metamaterials. Among these properties are the possibility of generating an effective magnetic response from a composite system comprising non-magnetic materials, due to finite-wavelength effects [14], and the ability of an array of perfectly conducting thin wires to give an electrical response alike to that of a free-electron plasma [15].

A similar field is the study of the optical properties of composite materials, which can be traced back to Egyptian and Roman times, when the technology of coloring glass by the addition of metallic particles to the melt was developed. This field became very active in the 1970 s and early 1980s, when the challenge was to develop structured materials capable of providing the efficient conversion of incident solar energy to heat. Similar techniques are still under development, although the current focus is more on efficient photovoltaic absorbers rather than photothermal absorbers. The main question in the study of optical composite materials is that of obtaining an effective relative dielectric permittivity $\varepsilon_{\text {eff }}$ of a set of metallic particles in a dielectric background material, given that the particles and background material have dielectric permittivities of $\varepsilon_{1}$ and $\varepsilon_{2}$, respectively. Three main approaches are used, which are described comprehensively by Milton [2]. The first is based on the Maxwell-Garnett formula, or its multipole variants, and makes $\varepsilon_{\text {eff }}$ a rational function with its poles and zeros on the negative axis of $\varepsilon_{1} / \varepsilon_{2}$. The pole associated with the dipole resonance gives the plasmon condition for isolated metallic particles. The second approach is based on effective-medium formulae of the type pioneered by Bruggeman, which give a formula for $\varepsilon_{\text {eff }}$ in the form of a square root and therefore have a branch cut on the negative axis of $\varepsilon_{1} / \varepsilon_{2}$. The third approach is based on a comprehensive set of bounds - the Bergman-Milton bounds-in which the order determines the number of poles and zeros of $\varepsilon_{\text {eff }}$ on the negative axis of $\varepsilon_{1} / \varepsilon_{2}$.

It should be noted that the method used to find $\varepsilon_{\text {eff }}$ can also be applied to the effective magnetic permeability $\mu_{\text {eff }}$ for a composite with magnetic contrast. The effective properties are used in Maxwell's equations, assuming the quasistatic approximation to be valid; this requires the particles to be much smaller than the wavelength of light, say by a factor of 5-10. The ideas underlying the effective properties of composite materials can also be used to design systems that deliver greatly enhanced electric fields in localized regions for applications such as sensing and surface-enhanced Raman scattering.

A problem in elasticity theory that may be likened to that of electromagnetic cloaking is the design of neutral inclusions. Neutral inclusions are inserts into elastic bodies that do not disturb the original stress distribution. They have been studied since 1953 [16] and continue to be of interest today, with their analogy to cloaking now fully recognized and exploited [17]. A similar study in electrical tomography (the detection of a conducting body's internal structure by the measurement of currents flowing in response to a pattern of applied voltages) was made by Greenleaf et al. [18]. These authors obtained what might be called 'conductivity-cloaked' systems in a way that parallels strongly independent (and later) developments in electromagnetic cloaking by Pendry, Schurig and Smith [7].

The final precursor to the era of metamaterials $[19,20]$ is marked by studies of the quasistatic properties of arrays of coated cylinders. Researchers identified that a cylinder composed of a core (radius $r_{c}$, dielectric permittivity $\varepsilon_{\mathrm{c}}$ ) and a shell $\left(r_{\mathrm{s}}\right.$, dielectric permittivity $\left.\varepsilon_{\mathrm{s}}\right)$, when placed in a matrix material of dielectric permittivity $\varepsilon_{\mathrm{m}}$, would have two possibilities for resonance. In the first, for $\varepsilon_{\mathrm{c}}+\varepsilon_{\mathrm{s}}=0$, the shell and core form a resonant combination and the effective permittivity of the array is exactly that of a solid cylinder with $\varepsilon_{\mathrm{c}}$ extending out to the shell radius $r_{\mathrm{s}}$. In the second, for $\varepsilon_{\mathrm{s}}+\varepsilon_{\mathrm{m}}=0$, the remarkable result is that the effective permittivity of the array is exactly that of a solid cylinder with $\varepsilon_{\mathrm{c}}$ extending out to the magnified radius $r_{\mathrm{s}}^{2} / r_{\mathrm{c}}$. 


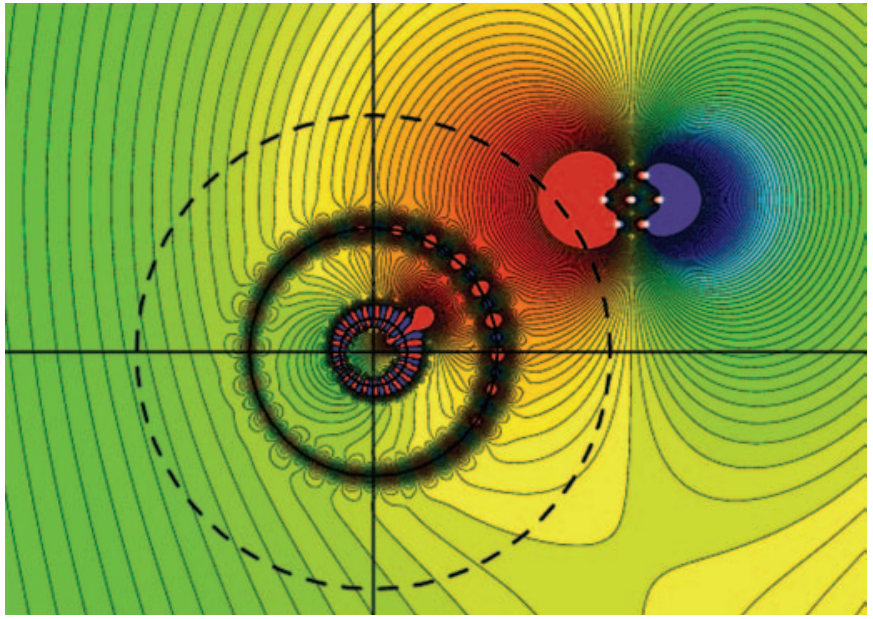

Figure 2. A cluster of seven polarizable dipoles about to enter the cloaking region (dashed circle) of a coated cylinder. Adapted from Ref. 22 (৫) 2007 OSA).

With the advent of research into cloaking, these results were taken up again $[21,22]$. It was discovered that these resonances could be used to provide external cloaking, in which a coated cylinder with a core of permittivity $\mathcal{E}_{\mathrm{c}}=\varepsilon_{\mathrm{m}}$ and a shell of permittivity $\varepsilon_{\mathrm{s}}=-\mathcal{E}_{\mathrm{m}}$ could cloak polarizable systems of dipoles placed in a region outside it. This process is illustrated in Figure 2, a frame from an animation by Nicorovici et al. [22]. Here, a cluster of seven polarizable dipoles placed in an external electric field is about to enter the cloaking region of a coated cylinder. The induced charges on the core-shell and shellmatrix boundaries are evident; these will create a reaction field that cancels out the applied field near each polarizable dipole, thus rendering the cluster invisible when in the cloaking region. A related type of external cloaking [23] uses the concept of complementary media, due to Pendry [4]. The work of Alu and Engheta [24] provides a bridge between the external cloaking ideas of Milton and co-workers and the literature on cloaking and neutral inclusions for electromagnetism and conductivity.

\section{Tunability of metamaterials}

Theoretical predictions [25-28] and experimental fabrication and measurements $[29,30]$ have both proved the possibility to create novel types of microstructured materials capable of demonstrating many unique properties not found in nature, including a negative index of refraction. One of the first realizations of such a composite material was created by a lattice of wires and split-ring resonators, which was shown to possess a negative real part of its magnetic permeability and dielectric permittivity at microwave frequencies. Figures $3(\mathrm{a}-\mathrm{d})$ show several examples of microwave metamaterials and metamaterial-based structures fabricated in Canberra for operation with microwaves. This includes metamaterial created by a square lattice of nonlinear split-ring resonators [31] (Figure 3(a)), a slab of nonlinear electric metamaterial composed of electric resonators [32] (Figure 3(b)), magnetic metamaterial based on cut-wire pairs [33], which allow us to create truly two-dimensional metamaterials suitable for scaling to optical frequencies (Figure 3(c)), and a cloaking device based on spiral elements (Figure 3(d)) similar to the structure suggested by Tretyakov et al. [34].

Metamaterials are prominent for the exceptional opportunities they offer in tailoring the macroscopic properties of materials through appropriate choice and arrangement of their structural elements $[35,36]$. In this way, it is not only possible to design a metamaterial for a required functionality, but also to implement further adjustment capabilities at the level of assembly. This makes metamaterials different from conventional materials and provides exciting opportunities for implementing tunability.
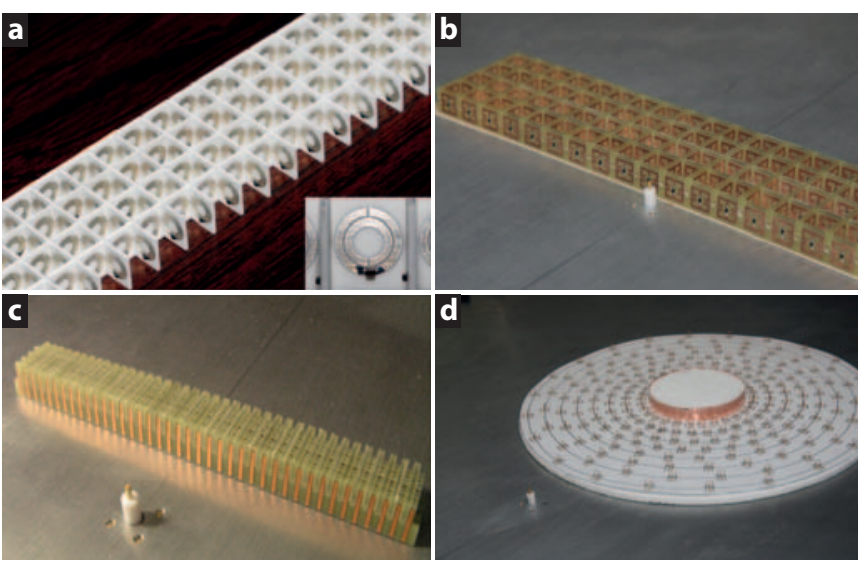

Figure 3. Examples of microwave metamaterial structures fabricated in Canberra. (a) Nonlinear tunable magnetic metamaterial created by a square lattice of nonlinear splitring resonators. Each split-ring resonator contains a varactor that provides a powerdependant nonlinear response. Adapted from Ref. 31 (๔ 2008 OSA). (b) Nonlinear electric metamaterials. Adapted from Ref. 32 (@ 2009 AIP). (c) Magnetic metamaterials based on cut-wire pairs. Adapted from Ref. 33 (๔ 2008 OSA). (d) Metamaterial cloaking device based on a lattice of spiral elements.

Tunable metamaterials have the ability to continuously change their properties in response to certain external influences or signals, with the mechanism of tunability being intrinsic to the metamaterial. The key means of tuning a resonant metamaterial lies in affecting the system so as to change the parameters of the resonance. The characteristics of the metamaterial can therefore be varied, enabling tunable transmission. Generally speaking, we can outline several different strategies to achieve tunability in metamaterials. The first of these is to modify a basic element of the composite structure, such as a split-ring resonator or a cut-wire pair. For microwaves, this is achieved by introducing a varactor diode as an externally or nonlinearly tunable element. This causes a shift in frequency of a split-ring resonator and, for a composite structure, allows either the magnetic or electric response (or both) to be controlled by changing the incident power [37-40]. The second technique is to select a tunable substrate that supports layers of elements such as split-ring resonators; one can employ the specific properties of the substrate to achieve a tunable response for the whole structure [41-43]. Similarly, the substrate may change its properties under external illumination. The third technique is to engineer the structural geometry of the metamaterial, which modifies near-field coupling between the elements - known as structural tunability [44,45]. The most straightforward lattice-tuning approach is to vary the lattice constant $b$. It was shown [46] that the resonance frequency can be remarkably shifted in this way, and this prediction was confirmed by microwave experiments [44]. Accordingly, a slab of metamaterial can be tuned between transmission, absorption, reflection, and then back to transmission. A clear disadvantage of this method is that significantly varying $b$ implies a corresponding change in the overall dimension of the metamaterial along $z$, which might be undesirable for certain applications.

To prove this concept of structural tunability, Lapine et al. [45] opted for a small reconfigurable system comprising single-split rings (2.25 mm mean radius, $0.5 \mathrm{~mm}$ strip width, $1 \mathrm{~mm}$ gap) printed with a period of $a=7 \mathrm{~mm}$ on $1.5 \mathrm{~mm}$-thick circuit boards. They used five resonators in the propagation direction $x$ and only one period along $y$; 30 boards were stacked together in the $z$ direction with a minimal possible lattice constant of $I=1.5 \mathrm{~mm}$ used for the measurements. The estimated resonance frequency of a single resonator (in a dielectric environment) was about $4.9 \mathrm{GHz}$, although the resonance of the dense metamaterial was significantly shifted to lower frequencies. To minimize the undesirable bianisotropic effects associated with singlesplit rings, the boards were assembled so that the gaps were oppositely 

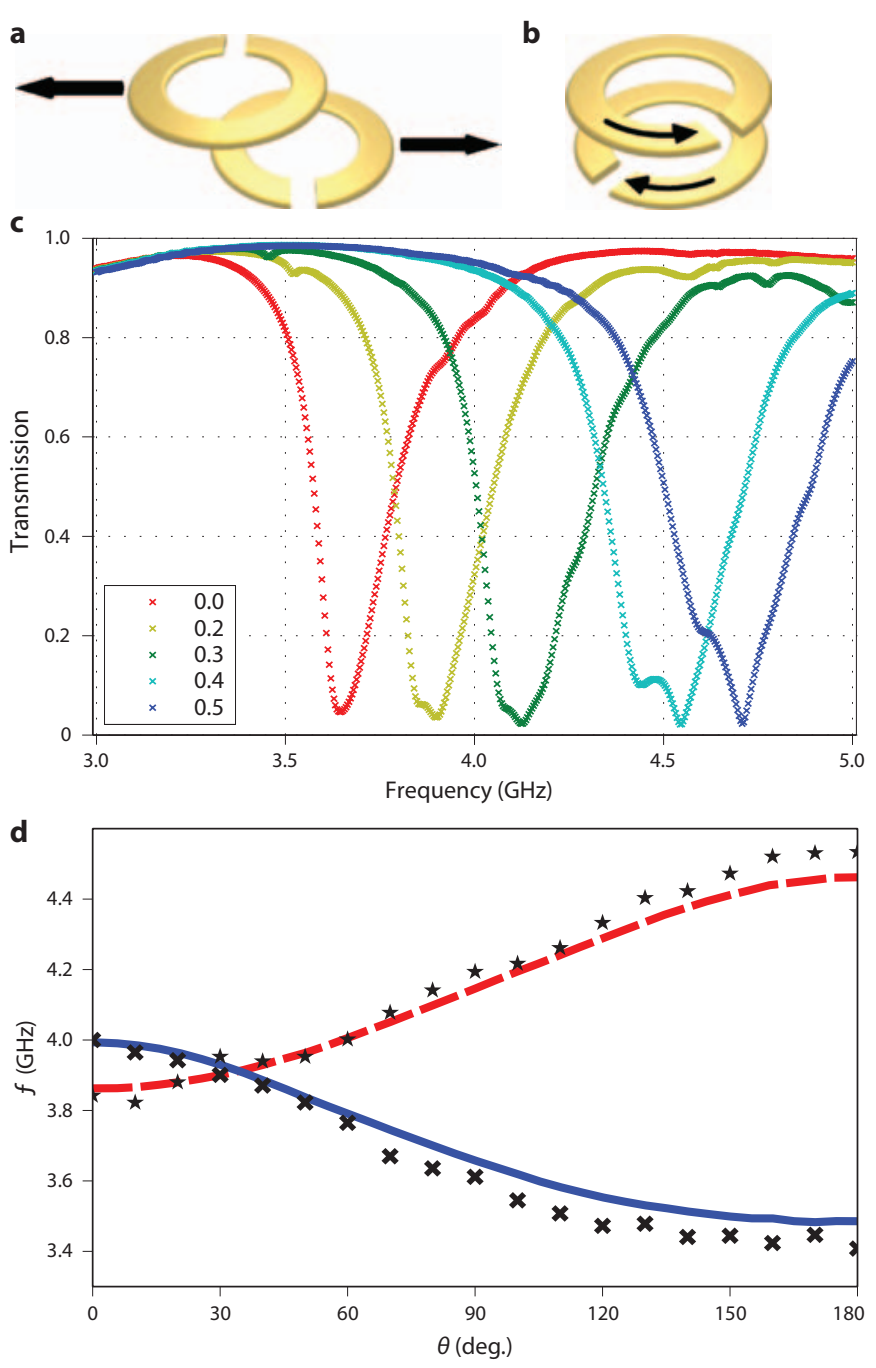

Figure 4. $(a, b)$ Two ways of structurally tuning the split-ring resonator interaction. $(c, d)$ Experimentally measured shift of magnetic resonances. Adapted from Refs 48 (๔ 2010 APS) and 84 (๔ 2011 APS). In (c), the curves with dips from left to right correspond to an increasing lattice shift [45].

oriented in adjacent layers, resembling the logic of broadside-coupled split-ring resonators [47]. Transmission measurements (using a Rohde and Schwarz ZVB Network Analyzer) were performed for various lattice shifts in a WR-229 rectangular waveguide.

The experimental transmission spectra of these measurements, shown in Figure 4, demonstrate excellent tuning of the resonance frequency. Furthermore, comparing the experimental resonance shift with theoretical predictions shows [45] that the experimental system demonstrates an even higher efficiency. This effect can be explained by accounting for mutual capacitance between the resonators, which is neglected in theoretical calculations. Indeed, for the broadside-like configuration of rings, mutual capacitance between the resonators is distributed along the whole circumference [47]. When the resonators are laterally displaced, the mutual capacitance decreases, and this effect adds to the increase of resonance frequency imposed by decreased inductive coupling.

Recently, Powell et al. [48] analyzed the near-field interaction between the resonant subwavelength elements of a metamaterial and presented a method for calculating the electric and magnetic interaction coefficients. They demonstrated that by adjusting the relative configuration of the neighboring split-ring resonators it becomes possible to manipulate this near-field interaction and therefore tune the response of the metamaterial. Powell et al. [48] used the results of this analysis to explain previous experimental observations when tuning microwave metamaterials [45].

\section{Nonlinearity in metamaterials}

The possibility of controlling the effective parameters of a metamaterial by exploiting the nonlinear response of split-ring resonators was suggested theoretically some time ago [49-51], but only recently have these ideas been implemented in a novel type of nonlinear tunable structure [31,52]. A similar approach can be employed for creating nonlinear electric metamaterials [32]. However, such methods become increasingly difficult to implement at higher frequencies. Some alternative approaches developed very recently employ an analogy with natural materials that demonstrate different properties depending on their specific crystalline structures [45]. Metamaterials allow us to realize great opportunities for designing unique structures with desired response functions and implementing convenient mechanisms for achieving tunability. More importantly, the range of tunability for a given property in a metamaterial can be much larger than in a natural material, as the lattice effects can be made much stronger through a higher efficiency of collective effects in the lattice, achieved by an appropriate design.

\section{Single nonlinear split-ring resonator}

Effective control over the resonant conditions of a split-ring resonator is achieved by adding the capacitance of a diode in series with the distributed capacitance of the outer ring of the resonator at a point of maximum in the electric current. The series application of the diode provides a simple mechanism for achieving both tunability and nonlinearity that is suited to the formation of metamaterials, particularly with recent developments in magnetic thin-film and microwave nonlinear materials. The symmetry and simplicity of such systems also lends them to greater integration, allowing the structure to be translated more readily to the terahertz and optical frequency domains. To study both the tunability and nonlinearity of metamaterials, Shadrivov et al. [37] used a single archetype split-ring resonator constructed on fibreglass (FR4, $\varepsilon_{\mathrm{r}}=4.4$ ) with copper metallization. The resonator had an internal ring radius of $2.56 \mathrm{~mm}$. Both rings had a linear width of $1.44 \mathrm{~mm}$, and the separations between the rings and ring slots were $0.32 \mathrm{~mm}$. For a negative bias voltage of $-10 \mathrm{~V}$ (not shown) the resonant frequency can be shifted to $2.9 \mathrm{GHz}$, whereas for a positive voltage of $1 \mathrm{~V}$ the resonant frequency decreases to $2.27 \mathrm{GHz}$. This particular varicap diode and split-ring resonator structure has a tuning bandwidth of $0.63 \mathrm{GHz}$, which is equivalent to a tuning range of approximately 26\%. Different tuning regimes can be achieved by adding an inductive coil in parallel with the varactor [38]. The coil can change the sign of the nonlinearity and thus eliminate the memory effect caused by charge accumulation across the varactor. In addition, at higher powers the nonlinear response of the split-ring resonator becomes multi-valued, which paves the way for creating bistable tunable metamaterials [38]. The varactor diode introduced in the splitring resonator alters the resonant frequency $\omega_{\mathrm{r}}$, giving

$$
\omega_{\mathrm{r}}=\omega_{0}\left(1+\frac{C_{\mathrm{SRR}}}{C_{\mathrm{eff}}}\right)^{1 / 2}
$$

Here, $C_{\text {eff }}$ and $C_{\mathrm{SRR}}$ are the capacitances of the varactor diode and splitring resonator, respectively, and $\omega_{0}=\left(L_{\mathrm{SRR}} C_{\mathrm{SRR}}\right)^{-1 / 2}$, where $L$ is conductance. Shadrivov et al. [37] found that the diode discharges very slowly without the coil. It was therefore necessary to scan gradually through the frequency range to ensure that the measurements at each frequency were not affected by the rectification occurring at another. The resonant frequency was determined from the minimum of the reflection coefficient. The configuration without the coil shows a stronger nonlinearity, but it has a pronounced memory effect that slows down the response to changes in the input power. Additionally, the resonator without the coil 
was found to be highly sensitive to interference from electrical wiring within the building [37]. This problem is largely eliminated once the resonator is placed inside the cavity. These results pave the way for the creation of nonlinear active metamaterials composed of tunable splitring resonators, as discussed below.

\section{Nonlinear magnetic metamaterials}

Nonlinear magnetic metamaterials operating at microwave frequencies can be fabricated by modifying the properties of split-ring resonators and introducing varactor diodes in each element of the composite structure $[37,38]$, such that the whole structure becomes dynamically tunable by varying the amplitude of the propagating electromagnetic waves. In this way, one can demonstrate the power-dependent transmission of the magnetic metamaterial at higher powers [31,52], as suggested earlier theoretically [49], thus experimentally realizing the nonlinearity-dependent enhancement or suppression of the transmission in a dynamically tunable magnetic metamaterial. Metamaterial samples (Figure 3(a)) were fabricated from $0.5 \mathrm{~mm}$-thick Rogers R4003 printed circuit boards with a nominal dielectric constant of 3.4. Shadrivov et al. [37] made dielectric boards containing the appropriate slot allocations with tin-coated copper nonlinear split-ring resonators. A photograph of one of several nonlinear metamaterial structures is shown in Figure 3(a). Each splitring resonator contains a variable-capacity diode (Skyworks SMV-1405) that introduces a nonlinear current-voltage dependence and results in a nonlinear magnetic dipole moment for each split-ring resonator [37]. In terms of effective medium parameters, the manufactured structure has both nonlinear magnetization and nonlinear effective magnetic permittivity [49]. Arrays of split-ring resonators formed a two-dimensional square lattice with $29 \times 4 \times 1$ unit cells, each measuring $10.5 \mathrm{~mm}$ in width.

To identify the effect of the nonlinearity, Shadrivov et al. [37] measured the transmission properties of the tunable magnetic metamaterial for different values of the input power. To measure the electromagnetic field scattering for the samples, the metamaterial slab was placed in a parallel plate waveguide. The planes of split-ring resonators were aligned perpendicular to the parallel plate surfaces. The input antenna, which consisted of a teflon-coated conductor measuring $1.26 \mathrm{~mm}$ in diameter and $11 \mathrm{~mm}$ in length, was placed at the midpoint of the lower plate, $2 \mathrm{~mm}$ from the metamaterial slab and in front of the central unit cell. The teflon coating improved the energy coupling into the waveguide for the wavelengths of interest. The antenna was positioned perpendicular to the bottom plate so that the excited electric field was polarized perpendicular to the plane, and thus parallel to the wires. The magnetic field of the wave was comprised primarily of an in-plane component that effectively excited the split-ring resonators. Close positioning of the source antenna to the metamaterial was chosen to funnel high electromagnetic power into the metamaterial sample for observing nonlinear effects. Shadrivov et al. [37] noted that although different positioning of the source antenna with respect to the central unit cell of the metamaterial gave slightly different quantitative results for the measured transmission, all the results were qualitatively identical. This effect appeared due to different antenna impedance matching to the sample. An identical antenna was placed in the center of the top plate and then used as a receiver for spectra measurements and for rasterscanning the electric field distribution in the horizontal plane. The input antenna was excited using an Agilent E8364A Vector Network Analyzer whose output was amplified by an HP 83020A $38 \mathrm{~dB}$ amplifier. For the transmission measurements, the receiving antenna, which was also connected to the network analyzer, was located $2 \mathrm{~cm}$ behind the metamaterial slab and in front of the central unit cell of the metamaterial. Measurements of the electric field inside the waveguide were evaluated in terms of the magnitude and phase of the transmission coefficient $S_{21}$ between the input of the source and the output of the receiver antenna. Due to the two-dimensional nature of the parallel plate waveguide, as well as the symmetry of the sample, the electric field in the scanned area was expected to remain polarized mainly perpendicular to the plane of the plates.

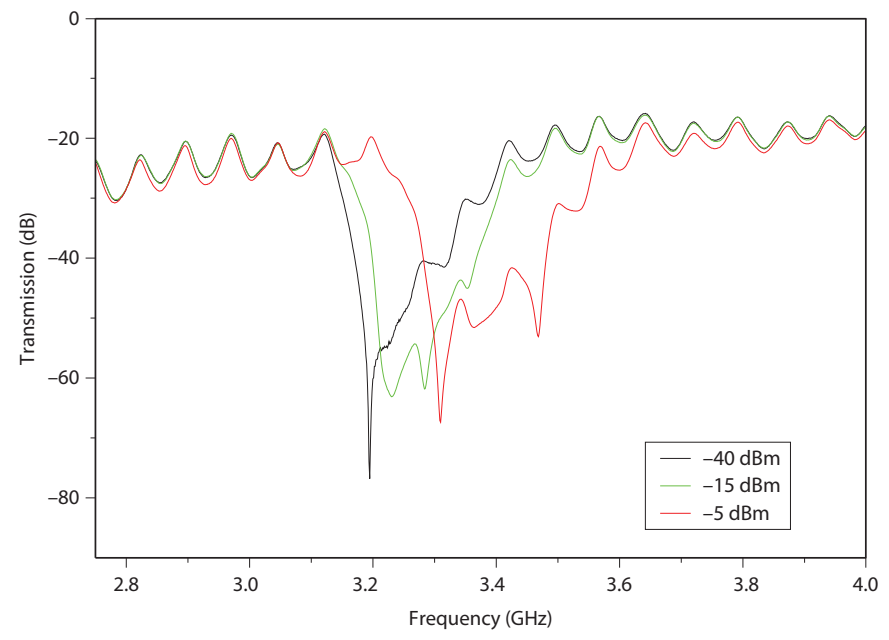

Figure 5. Experimentally measured transmission coefficients of a nonlinear magnetic metamaterial at different power levels. Adapted from Ref. 31 (๑ 2008 OSA).

To analyze the power-induced shift of the magnetic resonance due to the action of the varactor diodes introduced into the split-ring resonators, Shadrivov et al. [37] measured the transmission of the magnetic metamaterial for different values of the input power. Figure 5 shows the dependence of the transmission coefficient on the frequency for three different values of the input power. The resonant frequency was shifted to the right when the input power grew, in a manner similar to the nonlinearity-induced effects observed for a single split-ring resonator $[37,38]$. These results show that choosing the operational frequency to be near the resonance allows us to change the transmission properties of the metamaterial dynamically by varying the input power. If the parameters of a metamaterial vary and cross the boundary between positive and negative values of the effective magnetic permeability, structural properties such as transmission will be switched as well, so that the material itself will change from opaque to transparent. Because the intensity of electromagnetic waves generated by a point source is nonuniform, a shift of resonances for individual split-ring resonators is inhomogeneous inside the metamaterial structure. Resonators closer to the source will experience stronger fields and thus it is expected that only the central part of the metamaterial will become transparent. Experimental results confirmed this effect by revealing a narrow aperture of the beam emerging from the metamaterial [31].

In the same metamaterial sample, Shadrivov et al. [37] observed the opposite effect when the transmission was suppressed by the nonlinearity at the high-frequency side of the resonance. Although the metamaterial was transparent for low powers, the growth of the wave amplitude made part of the metamaterial opaque and therefore prevented the radiation from propagating through the sample.

\section{Nonlinear electric metamaterials}

Given that a nonlinear shift of the resonance results in a very strong nonlinear magnetic response for split-ring resonators, Powell et al. [32] took an approach similar to the design of nonlinear electric resonators to demonstrate a strong nonlinear electric response [32]. Their structure is shown in Figure 3(b), in which two perpendicular sets of boards have been introduced to create a relatively isotropic response. Within each resonator, an additional gap was introduced and a varactor diode placed inside, which provides an additional series capacitance for tuning the resonant frequency. The lattice period was $11 \mathrm{~mm}$, and the resonators were fabricated on copper-clad FR 4 with a width and height of $8 \mathrm{~mm}$, a track width of $1 \mathrm{~mm}$, outer gaps of $0.4 \mathrm{~mm}$ separation and a length of $2.4 \mathrm{~mm}$.

Powell et al. [32] measured the transmission response at incident powers of 10, 20 and $30 \mathrm{dBm}$, as shown in Figures $6(\mathrm{a}-\mathrm{c})$. At the lowest incident power $(10 \mathrm{dBm})$, there was negligible tuning of the response by the incident wave, and thus the transmission response in this case 

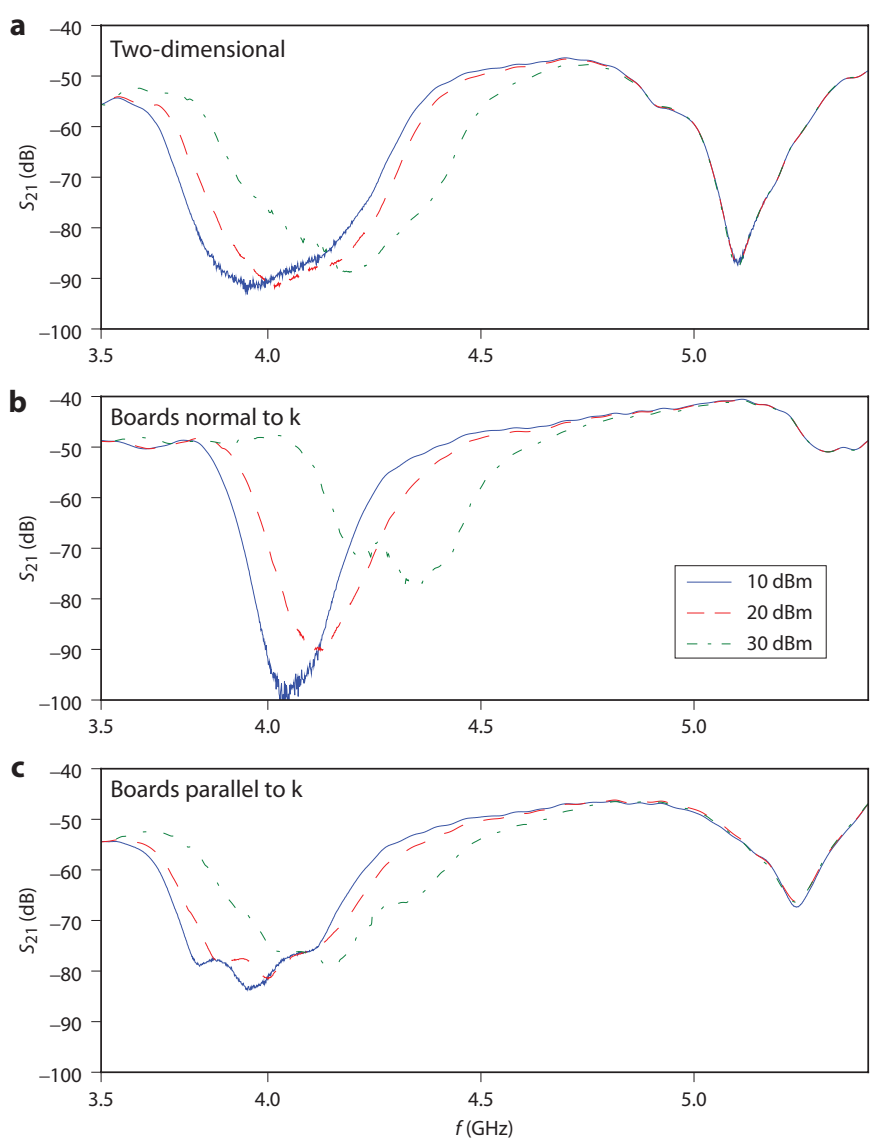

Figure 6. $(\mathrm{a}-\mathrm{c})$ Experimental nonlinear transmission response at incident powers of 10, 20 and $30 \mathrm{dBm}$ for different orientations of a nonlinear electric metamaterial. Adapted from Ref. 32 (@ 2009 AIP).

was essentially linear. The large insertion loss away from resonance was due to the deliberately mismatched receiving probe, which was designed for minimal perturbation of the fields within the structure. Figure 6(a) shows that the higher-frequency mode did not shift its frequency with a change in incident power. This mode consisted of two current loops flowing in the same direction, whose magnetic dipole moments add constructively. The accumulated charges across the gaps were travelling in opposite directions and therefore resulted in a vanishing electric dipole moment. As there was no net current through the central conductor, the nonlinear response of the varactor diode did not come into play. These mode configurations, and the stop-band locations, were confirmed by numerical simulations of transmission performed in CST Microwave Studio using a single element with electric boundaries in the vertical direction and magnetic boundaries in the horizontal direction.

For comparison, Powell et al. [32] individually investigated the two different circuit board orientations (normal or parallel to the direction of propagation). The nonlinear transmission responses for these structures are shown in Figures 6(b) and (c), respectively. A significant nonlinear response occurred in both cases. When the boards were perpendicular to the direction of propagation, the higher-frequency magnetic stop-band did not exist. This is due to the symmetry of the fields across the gaps and the lack of any magnetic field component normal to the rings. We note that because the experiment used a cylindrically symmetric source, there was some component of the wavenumber normal to the nominal propagation direction, and hence some vestige of the second resonance remained. Also worth noting is the fact that both resonances were noticeably modified in the isotropic configuration, compared with when they were measured separately. This is likely to be due to the strong electrical interaction between the nearest-neighbor boards in the orthogonal directions, as their gaps were in close proximity.

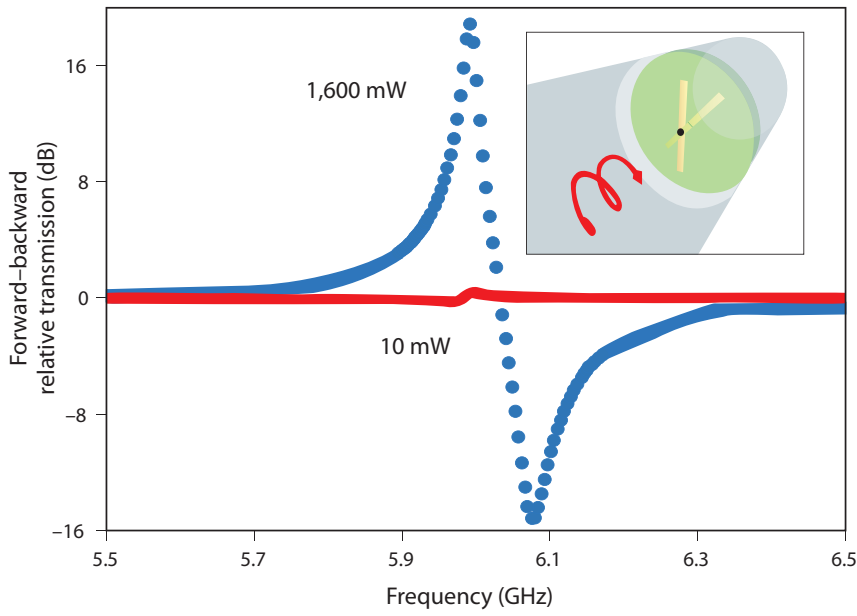

Figure 7. Asymmetric transmission properties of a single-chiral metamolecule. The ratio between the transmission coefficients in the forward and backward directions is shown in decibels. For low powers (red curve), the transmission is equivalent in both directions for left-handed circularly polarized waves. For high powers (blue curve), the transmission is strongly asymmetric. Adapted from Ref. 53 (@ 2011 IOP).

\section{Nonlinear chiral metamaterials}

The next idea is to combine strong chirality and the nonlinearity of metamaterials to develop a structure with nonlinear optical activitya polarization rotation that depends on the strength of the incident field [53]. This effect is almost negligible in natural crystals and has therefore not yet been used for real-life applications. Using metamaterials, we can achieve this effect by engineering the chiral response and carefully placing nonlinear elements within the structure. Such a metamaterial is designed to operate at microwave frequencies and consists of a pair of metallic wires, twisted so that they are no longer parallel, as shown in the inset of Figure 7 . We can see that the structure is chiral because its mirror image is not identical to the original. Nonlinearity is introduced by cutting each wire and inserting a varactor diode. Further details of the design and the experimental techniques can be found in Ref. 53. The response of the structure exhibits a strongly resonant feature caused by the excitation of currents in the left-handed metamolecule by the left-handed circularly polarized wave. At the same time, the right-handed circularly polarized wave does not noticeably excite any resonances in the structure. Changing the power of the incident wave shifts the resonance of the gyrotropic response to a higher frequency. Importantly, such a shift of the polarization rotation resonance leads to a giant nonlinear gyrotropy. Experimental results indicate a peak value of $15 \mathrm{deg} \mathrm{W}^{-1}$, which is 12 orders of magnitude stronger than results previously observed for $\mathrm{LiIO}_{3}$ at optical wavelengths.

Modifying the structure such that only one wire contains a nonlinear inclusion will lower the symmetry of the system, and we can use this feature to allow the propagation of left-handed circularly polarized waves in only one direction — an electromagnetic diode regime. Measurements for a left-handed circularly polarized wave scattering on a left-handed asymmetric chiral metamolecule are shown in Figure 7. When the amplitude of the incident wave is small, the structure exhibits a linear response and the transmission coefficients in both directions are equal. However, in the nonlinear regime a high-intensity incident wave causes considerably different transmission properties in opposite directions, with the maximal intensity contrast between the two directions being $18 \mathrm{~dB}$.

\section{Nonlinear parametric interactions}

Aside from the possibility of tuning the response of nonlinear metamaterials using various inclusions, a range of new parametric nonlinear phenomena were predicted and even demonstrated experimentally in composite structures, including novel phase-matching schemes for wave mixing. The most notable of these is the 'nonlinear optical mirror' [54], 
in which a nonlinear negative-index medium emits the generated frequency towards the source of the pump. The use of optical parametric amplification was suggested as a possible remedy to the problem of losses in metamaterials [55].

The unique phase-matching schemes available for metamaterials allow us to achieve parametric amplification in a cavity-free configuration, whereby pumping of the strongly attenuated backward wave is performed by a higher-frequency forwards pump wave. Although a wide range of nonlinear processes have already been demonstrated in nonlinear metamaterials, including frequency generation, parametric amplification and bistability, the experimental set-ups have so far been constrained to subwavelength interaction lengths for fear of the destructive effects of phase mismatch. As such, the achieved conversion efficiencies are only a fraction of their potential. However, phase matching is an exciting subject that involves the entire set of linear and nonlinear properties, bringing to bear the full capabilities of nonlinear metamaterials to manipulate light at will. Using this approach, the theoretical concept of a nonlinear optical mirror that generates and sends the second harmonic of incident radiation towards the fundamental frequency source was recently demonstrated experimentally in a bulk negative-index nonlinear metamaterial [56], along with two other novel phase-matching configurations, utilizing periodic poling to switch between the three phase-matching domains.

\section{Transformation optics across the spectrum}

The ideas of transformation optics are very general, extending across a wide variety of different wave types and frequency regions. A recent review article [57] surveyed the different wave types and governing equations for which transformation optics has been considered: chiefly water waves [58], plasmonics [59] and elastodynamic waves (phonons [60], platonics [61] and acoustics [62]). We recall our comment in the Introduction that some features of metamaterials were indeed anticipated in the literature on elastic waves [10].

Even in the context of Maxwell's equations, scale-invariance guarantees that basic ideas can be applied from the ultraviolet region (wavelengths of around $300 \mathrm{~nm}$ ) through to the radio-wave region (wavelengths in the meter range). What of course distinguishes each different wavelength region is the ease with which structures can be fabricated, the choice of materials available, the ability to probe electromagnetic fields within the structures and the range of applications for the final devices.

Much attention is currently being devoted to metamaterials for the visible and near infrared regions, with one major preoccupation being to overcome the effects of metallic loss [63-65]. However, we stress that interesting materials such as restrahlen crystals [66] exist in the mid-infrared region. We have also seen that many interesting concepts relating to linear and nonlinear metamaterials can be tested and implemented in the microwave region. Much of the electromagnetic spectrum is therefore suitable for the implementation of transformation optics, and is indeed already under active investigation.

\section{Metamaterials: making a short story long}

The reality of metamaterials and their huge potential for novel applications have been demonstrated in a number of experiments that span from radiowaves all the way to visible light. The fabrication techniques for metamaterials in these experiments depend largely on the wavelength of operation. For radio- and microwaves, individual resonators are millimeters or even centimeters in size, and can readily be made using printed circuit board technology. However, creating 3D materials requires a large number of such boards to be assembled. Printed circuit boards can still be used for planar terahertz metamaterials, where resonators are tens to hundreds of micrometers, but volumetric assemblies become more difficult to achieve in this regime. In the infrared and visible range, however, higher precision fabrication tools are required-a topic

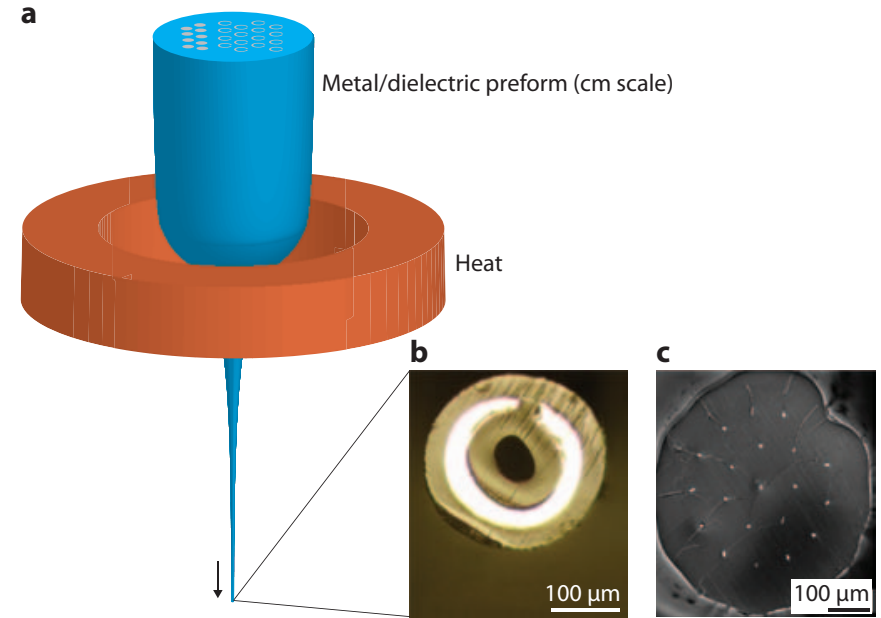

Figure 8. (a) Schematic of a metal-dielectric preform drawn into a metamaterial using fiber-drawing technology. (b) Scanning electron microscopy image of the cross-section of a fabricated indium slotted cylinder with a magnetic resonance in

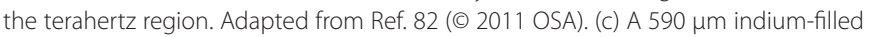
poly(methyl methacrylate) fiber, with plasma frequency in the terahertz region. Adapted from Ref. 75 (๔ 2010 AIP)

recently reviewed in Ref. 67. Metamaterials with a single or few layers of resonators can be produced using electron beam, focused ion beam and nano-imprinting lithography, or even directional evaporation techniques; fully three-dimensional metamaterials can be fabricated using direct laser writing with non-diffraction-limited photopolymerization (using stimulated emission depletion schemes, for example) followed by full or partial metallization-a versatile but somewhat slow process that can deliver only one small sample at a time. The self-assembly of metallic clusters, as well as using nanostructured porous materials as templates, can also yield unusual magnetic and electric properties. Of all these, it seems only self-assembly can lead to the large-scale fabrication of volumetric metamaterials, but with limited geometries and regularity.

It is progress in a different field of optics - the study of photonic crystal fibers [68] - that sparked the idea of an alternative technique suitable for the mass-production of metamaterials. Photonic crystal fibers are optical fibers (typically strands of glass or polymer) thinner than a human hair that contain arrays of micrometer-sized holes. They are fabricated by first assembling a macroscopic preform, in which holes are of the order of $1 \mathrm{~mm}$ in size. The preform is then heated and stretched in a furnace, during which the glass softens and is drawn like cooked sugar (Figure 8). During this drawing process, the cross-section of the preform can be preserved so that the array of holes is simply scaled down to microscopic or even nanoscopic dimensions. A very similar process known as the Taylor-wire process has been used to fabricate individual microscopic metallic wires for almost a century [69,70]. Irregular arrays of multiple thin metallic wires embedded in glass have also been demonstrated by drawing down metallic powders encapsulated in glass [71]. Bringing these two processes together, fibers with regular arrays of microscopic metallic wires were demonstrated at the University of Bath in 2008 [72], and almost simultaneously using a slightly different process at the University of Erlangen [73]. The direct-drawing process lends itself well to mass-fabrication, as a single macroscopic preform machined by conventional means can produce kilometers of fibers containing arrays of microscopic wires.

Wire arrays are one of the most basic metamaterials; their effective dielectric permittivity along the wires can be tuned between that of a metal and that of a dielectric, and they can exhibit low-frequency plasmon resonances [25]. Wire arrays can also be made to have extreme birefringence, whereby they behave as a metal in one direction and as a dielectric in another-a useful property for devices such as hyperlenses [74]. Tuniz et al. were the first to use fiber-drawing techniques 
to demonstrate electric metamaterials [75]. Their fibers comprised a hexagonal array of indium wires measuring $10 \mu \mathrm{m}$ in diameter in a polymer fiber, and were designed to operate at terahertz frequencies. Shortly after, Mazhorova et al. demonstrated the integration of similarly drawn metamaterials into a substrate [76]. Operation at infrared or visible frequencies will require thinner wires made out of low-loss metals such as silver or gold. Tyagi et al. [77] have recently shown that gold wires with diameters of the order of $100 \mathrm{~nm}$ can be included in optical fibers using the direct-drawing technique, and hole diameters down to $30 \mathrm{~nm}$ have also been demonstrated. Badinter et al. have successfully drawn arrays comprising many thousands of metal wires with individual diameters measuring just a few tens of nanometers [78]. At such dimensions, metamaterials could operate in the visible spectrum, and indeed Tuniz et al. have shown that a regular array of $30 \mathrm{~nm}$-diameter silver wires could achieve the dream of true optical invisibility, at least over a particular range of wavelengths and angles [79].

Although arrays of wires are indeed valuable, they do have limitations. In particular, they can suffer from strong spatial dispersion [80] and offer next to no control over the magnetic properties of the material. The latter could be achieved using non-circularly symmetric metallic inclusions in the fiber geometry $[81,82]$, but such longitudinally invariant magnetic resonators would also suffer from spatial dispersion. Patterning along the length of the metamaterial could alleviate both problems, thus providing real three-dimensional metamaterials. Designing metamaterials for terahertz and infrared light will require laser-machining using pulsed lasers to interrupt wires at appropriate length scales during the drawing process, whereas for the length scales required for the visible spectrum one could potentially make use of phase separation during the drawing process to achieve longitudinal patterning down to tens of nanometers [83].

\section{Conclusions}

We have discussed the main concepts of metamaterials, including the methods of fabrication, properties of the composites that allow simultaneous control of electric and magnetic fields through structuring on a scale much smaller than the wavelength of light, and optical effects achieved using metamaterial-based systems, such as backward wave propagation and negative refraction. Metamaterials are prominent for the exceptional opportunities they offer in tailoring the macroscopic properties of materials through appropriate choice and arrangement of their structural elements. In this way, it is not only possible to design a metamaterial for a required functionality, but also to implement further adjustment capabilities at the level of assembly. This makes metamaterials different from conventional materials and opens exciting opportunities for implementing tunability. We have discussed several ideas for implementing the tunability of metamaterials by tailoring the near-field interaction between the structured elements and by employing nonlinear elements. We have also emphasized the recent development of this field in Australia. In particular, we have discussed several striking conceptual and technological advances made in this rapidly developing field, including cloaking, nonlinear metamaterials and drawable metamaterials.

\section{Acknowledgments}

This work was supported by the Australian Research Council through its Discovery Grants and Centers of Excellence Programs. We thank our numerous collaborators for useful collaborations and many valuable comments.
9. A. Moroz, http://www.wave-scattering.com/negative.html (2009).

10. H. Lamb, P. Lond. Math. Soc. 1, 473 (1904).

11. A. Schuster, An Introduction to the Theory of Optics (Edward Arnold, 1904).

12. L. I. Mandelshtam, Zh. Eksp. Teor. Fiz. 15, 475 (1945).

13. L. S. Dolin, Izv. Vyssh. Uchebn. Zaved. Radiofiz. 4, 964 (1961) (in Russian).

14. L. Lewin, P. I. Electr. Eng. 94, 65 (1947).

15. W. Rotman, IEEE T. Antenn. Propag. 10, 82 (1962).

16. E. H. Mansfield, Q. J. Mech. Appl. Math. 6, 370 (1953).

17. S. Guenneau, A. Movchan, F. Zolla, N. Movchan, A. Nicolet, J. Comput. Appl. Math. 234, 1962 (2010).

18. A. Greenleaf, M. Lassas, G. Uhlmann, Math. Res. Lett. 10, 685 (2003).

19. N.A. Nicorovici, R. C. McPhedran, G. W. Milton, P. R. Soc. London A 442, 599 (1993).

20. N. A. Nicorovici, R. C. McPhedran, G. W. Milton, Phys. Rev. B 49, 8479 (1994)

21. G.W. Milton, N.A. Nicorovici, P. R. Soc. London A 462, 3027 (2006).

22. N. A. Nicorovici, G. W. Milton, R. C. McPhedran, L. C. Botten, Opt. Express 15, 6314 (2007).

23. Y. Lai, H. Chen, Z.-Q. Zhang, C. T. Chan, Phys. Rev. Lett. 102, 093901 (2009).

24. A. Alù, N. Engheta, J. Opt. A-Pure Appl. Op. 10, 093002 (2008).

25. J. B. Pendry, A. J. Holden, W. J. Stewart, I. Youngs, Phys. Rev. Lett. 76, 4773 (1996).

26. J. B. Pendry, A. J. Holden, D. J. Robbins, W. J. Stewart, IEEE T. Microw. Theory 47, 2075 (1999).

27. P. Markos,C. M. Soukoulis, Phys. Rev. E 65, 036622(2002).

28. P. Markos,C. M. Soukoulis, Phys. Rev. B 65, 033401 (2002).

29. D. R. Smith, W. Padilla, D. C. Vier, S.C. Nemat-Nasser, S. Schultz, Phys. Rev. Lett. 84, 4184 (2000).

30. C. G. Parazzoli, R. B. Greegor, K. Li, B. E. C. Koltenbah, M. Tanielian, Phys. Rev. Lett. 90, 107401 (2003).

31. I. V. Shadrivov, A. B. Kozyrev, D. W. van der Weide, Y. S. Kivshar, Opt. Express 16, 20266 (2008).

32. D. A. Powell, I. V. Shadrivov, Y. S. Kivshar, Appl. Phys. Lett 95, 084102 (2009).

33. D. A. Powell, I. V. Shadrivov, Y. S. Kivshar, Opt. Express 16, 15185 (2008).

34. K. Guven, E. Saenz, R. Gonzalo, E. Ozbay, S. Tretyakov, New J. Phys. 10, 115037 (2008).

35. M. Lapine, S. Tretyakov, IET Microw. Antenna P. 1, 3 (2007).

36. A. Sihvola, Metamaterials 1, 2 (2007).

37. I. V. Shadrivov, S. K. Morrison, Y. S. Kivshar, Opt. Express 14, 9344 (2006).

38. D. A. Powell, I. V. Shadrivov, Y. S. Kivshar, M. V. Gorkunov, Appl. Phys. Lett. 91, 144107 (2007).

39. B. Wang, J. Zhou, T. Koschy, C. M. Soukoulis, Opt. Express 16, 16058 (2008).

40. K. A. Boulais et al., Appl. Phys. Lett. 93, 043518 (2008).

41. S. O'Brien, D. McPeake, S. A. Ramakrishna, J. B. Pendry, Phys. Rev. B 69, 241101 (2004).

42. Z. Sheng,V. V. Varadan, J. Appl. Phys. 101, 014909 (2007).

43. N.-H. Shen et al., Phys. Rev. B 79, 161102(R) (2009).

44. I. V. Shadrivov, D. A. Powell, S. K. Morrison, Y. S. Kivshar, G. N. Milford, Appl. Phys. Lett. 90, 201919 (2007).

45. M. Lapine, et al., Appl. Phys. Lett. 95, 084105 (2009).

46. M. Gorkunov, M. Lapine, E. Shamonina, K. H. Ringhofer, European Physical Journal B 28, 263 (2002).

47. R. Marques, F. Mesa, J. Martel, F. Medina, IEEE T. Antenn. Propag. 51, 2572 (2003).

48. D. A. Powell, M. Lapine, M. V. Gorkunov, I. V. Shadrivov, Y. S. Kivshar, Phys. Rev. B 82, 155128 (2010).

49. A. A. Zharov, I. V. Shadrivov, Y. S. Kivshar, Phys. Rev. Lett. 91, 037401 (2003).

50. M. Lapine, M. Gorkunov, K. H. Ringhofer, Phys. Rev. E 67, 065601 (2003).

51. M. Gorkunov, M. Lapine, Phys. Rev. B 70, 235109 (2004).

52. I. V. Shadrivov, A. B. Kozyrev, D. W. van der Weide, Y. S. Kivshar, Appl. Phys. Lett. 93, 161903 (2008).

53. I. V. Shadrivov, V. A. Fedotov, D. A. Powell, Y. S. Kivshar, N. I. Zheludev, New J. Phys. 13, 033025 (2011).

54. I. V. Shadrivov, A. A. Zharov, Y. S. Kivshar, J. Opt. Soc. Am. B 23, 529 (2006).

55. A. K. Popov, V. M. Shalaev, Opt. Lett. 31, 2169 (2006).

56. A. Rose, D. Huang, D. R. Smith, Phys. Rev. Lett. 107, 063902 (2011).

57. S. Guenneau et al., J. Opt. 13, 024014 (2011).

58. M. Farhat, S. Enoch, S. Guenneau, A. B. Movchan, Phys. Rev. Lett. 101, 134501 (2008).

59. J. Renger et al., Opt. Express 18, 15757 (2010).

60. S. B. Platts. N. V. Movchan, R. C. McPhedran, A. B. Movchan, P. Roy. Soc. A 458, 2327 (2002).

61. M. Farhat, S. Guenneau, S. Enoch, Phys. Rev. Lett. 103, 024301 (2009).

62. S. Yang et al., Phys. Rev. Lett. 93, 024301 (2004).

63. K. Li, M. I. Stockman, D. J. Bergman, Phys. Rev. Lett. 91, 227402 (2003). 
64. M. A. Noginov et al., Nature 460, 1110 (2009).

65. R. F. Oulton et al., Nature 461, 629 (2009).

66. B. Neuner et al., Opt. Lett. 34, 2667 (2009).

67. C. M. Soukoulis,M. Wegener, Nature Photon., published online 17 July 2011 (DOI: 10.1038/nphoton.2011.154).

68. J. C. Knight, Nature 424, 847 (2003).

69. G. F. Taylor, Phys. Rev. 23, 655 (1924).

70. I. W. Donald, J. Mater. Sci. 22, 2661 (1987).

71. J. D. Ayers, J. Mater. Sci. 28, 2337 (1993).

72. J. Hou et al., Opt. Express 16, 5983 (2008).

73. M. A. Schmidt, L. N. Prill Sempere, H. K. Tyagi, C. G. Poulton, P. St. J. Russell, Phys. Rev. B 77, 033417 (2008).

74. Z. Jacob, L. V. Alekseyev, E. Narimanov, Opt. Express 14, 8247 (2006).
75. A. Tuniz et al., Appl. Phys. Lett. 96, 191101 (2010).

76. A. Mazhorova et al., Opt. Express 18, 24632 (2010).

77. H. K. Tyagi et al., Opt. Lett. 35, 2573 (2010).

78. E. Badinter, A. Ioisher, E. Monaico, V. Postolache, I. M. Tiginyanu, Mater. Lett. 64, 1902 (2010).

79. A. Tuniz, B. T. Kuhlmey, S. Fleming, Opt. Express 18, 18095 (2010).

80. P. Belov et al., Phys Rev. B 67, 113103 (2003).

81. A. Wang et al., Opt. Mater. Express 1, 115 (2011).

82. A. Tuniz et al., Opt. Express 19, 16480 (2011).

83. K. Han, J. D. Embury, J. J. Petrovic, G. C. Weatherly, Acta Mater 46, 4691 (1998).

84. D. A. Powell, K. Hannam, I. V. Shadrivov, Y. S. Kivshar, Phys. Rev. B 83, 235420 (2011).

\section{Author profiles}

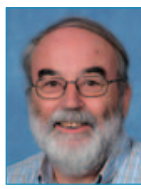

\section{Ross McPhedran}

Ross McPhedran received his PhD in 1973 from the University of Tasmania, Australia, after which he took up a postdoctoral fellowship in Marseille, France, for a year. He then moved to the University of Sydney, Australia, where he has remained since, apart from periods of study leave at Caltech, the Courant Institute, the universities of Bath and Liverpool, and the Université Paul Cezanne. He holds a Personal Chair in Electromagnetic Physics. His research interests include electromagnetic scattering by structured systems, composite materials, plasmonics, photonic and phononic crystals, and metamaterials.

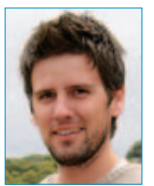

\section{Boris Kuhlmey}

Boris Kuhlmey received his PhD jointly from the Université Paul Cézanne, France, and the University of Sydney, Australia, in 2003. He has since remained at the University of Sydney, where he currently holds a senior research fellowship. His research interests include photonic crystal fibers, biophotonics, plasmonics and metamaterials.

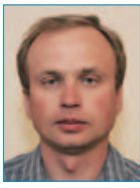

\section{Ilya Shadrivov}

Ilya Shadrivov received his PhD in physics in 2005 from the Australian National University. Since 2005 he has held several research positions at the Research School of Physics and Engineering of the Australian National University, where presently he is Queen Elizabeth II Fellow at the Nonlinear Physics Centre. His research interests include nonlinear and tunable metamaterials, light localization in disordered structures, and photonic crystals.

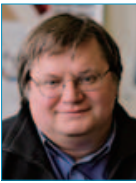

\section{Yuri Kivshar}

Yuri Kivshar received his PhD in 1984 from the USSR Academy of Science and then moved to the Institute for Low Temperature Physics and Engineering in Kharkov, Ukraine. From 1988 to 1993 he worked at different research centers in the USA, France, Spain and Germany. In 1993, he accepted a research position at the Research School of Physics and Engineering of the Australian National University, where presently he is a research professor and head of the Nonlinear Physics Center. His research interests include nonlinear waves, solitons, photonic crystals, metamaterials and nanophotonics. 